Article

\title{
Serum Levels of the Cytokine TWEAK Are Associated with Metabolic Status in Patients with Prostate Cancer and Modulate Cancer Cell Lipid Metabolism In Vitro
}

\author{
Antonio Altuna-Coy ${ }^{1,+}$, Xavier Ruiz-Plazas ${ }^{1,2,+}$, Marta Alves-Santiago ${ }^{1,2}$, José Segarra-Tomás $1,2, *, \ddagger$ \\ and Matilde R. Chacón ${ }^{1, *, \ddagger}$ \\ 1 Disease Biomarkers and Molecular Mechanisms Group, IISPV, Joan XXIII University Hospital, Universitat \\ Rovira i Virgili, 43007 Tarragona, Spain; antonio.altuna@iispv.cat (A.A.-C.); xruiz.hj23.ics@gencat.cat (X.R.-P.) \\ malves.hj23.ics@gencat.cat (M.A.-S.) \\ 2 Urology Unit, Joan XXIII University Hospital, 43005 Tarragona, Spain \\ * Correspondence: jsegarra.hj23.ics@gencat.cat (J.S.-T.); matilde.rodriguez@urv.cat (M.R.C.); \\ Tel.: +34-977295500 (ext. 3406) (J.S.-T. \& M.R.C.) \\ + These authors contributed equally to this work. \\ $\ddagger \quad$ These authors are joint senior authors on this work.
}

check for

updates

Citation: Altuna-Coy, A.; Ruiz-Plazas, X.; Alves-Santiago, M.; Segarra-Tomás, J.; Chacón, M.R. Serum Levels of the Cytokine TWEAK Are Associated with Metabolic Status in Patients with Prostate Cancer and Modulate Cancer Cell Lipid Metabolism In Vitro. Cancers 2021, 13, 4688. https: / / doi.org/10.3390/cancers13184688

Academic Editor: Frédéric Bost

Received: 20 August 2021

Accepted: 14 September 2021

Published: 18 September 2021

Publisher's Note: MDPI stays neutral with regard to jurisdictional claims in published maps and institutional affiliations.

Copyright: (c) 2021 by the authors. Licensee MDPI, Basel, Switzerland. This article is an open access article distributed under the terms and conditions of the Creative Commons Attribution (CC BY) license (https:/ / creativecommons.org/licenses/by/ $4.0 /)$.
Simple Summary: TWEAK is an inflammatory cytokine related to prostate cancer (PCa) progression that exerts its effects by engaging its cognate receptor Fn14. A soluble form of TWEAK (sTWEAK) has been detected in the PCa microenvironment. Altered levels of circulating sTWEAK are associated with aberrant glucose metabolism. We show that reduced serum levels of sTWEAK are associated with the metabolic status in patients with PCa and that the treatment of PC-3 cells with sTWEAK enhances the expression of genes related to lipid, but not to glucose, metabolism. sTWEAK also increases the lipid uptake and lipid accumulation in PC-3 cells. We corroborated that the observed effects were due to TWEAK/Fn14 engagement by silencing Fn14 expression, which attenuated the aberrant gene and protein expression. Additionally, we observed that the phosphorylation of ERK1/2 and AKT (ser473) were required for TWEAK/Fn14 actions. Thus, the contribution of the sTWEAK/Fn14 axis on PCa metabolism supports its potential as a therapeutic target for PCa.

Abstract: Soluble TWEAK (sTWEAK) has been proposed as a prognostic biomarker of prostate cancer (PCa). We found that reduced serum levels of sTWEAK, together with higher levels of prostate-specific antigen and a higher HOMA-IR index, are independent predictors of PCa. We also showed that sTWEAK stimulus failed to alter the expression of glucose transporter genes (SLC2A4 and SLC2A1), but significantly reduced the expression of glucose metabolism-related genes (PFK, HK1 and PDK4) in PCa cells. The sTWEAK stimulation of PC-3 cells significantly increased the expression of the genes related to lipogenesis (ACACA and FASN), lipolysis (CPT1A and PNPLA2), lipid transport (FABP4 and CD36) and lipid regulation (SREBP-1 and PPARG) and increased the lipid uptake. Silencing the TWEAK receptor (Fn14) in PC-3 cells confirmed the observed lipid metabolic effects, as shown by the downregulation of ACACA, FASN, CPT1A, PNPLA2, FABP4, CD36, SREBP-1 and PPARG expression, which was paralleled by a reduction of $F A S N, C P T 1 A$ and $F A B P 4$ protein expression. Specific-signaling inhibitor assays show that ERK1/2 and AKT (ser473) phosphorylation can regulate lipid metabolism-related genes in PCa cells, pointing to the AKT locus as a possible target for PCa. Overall, our data support sTWEAK/Fn14 axis as a potential therapeutic target for PCa.

Keywords: TWEAK; prostate cancer; lipid metabolism; Fn14

\section{Introduction}

Prostate cancer (PCa) is the second-most commonly diagnosed cancer in men worldwide [1]. A central challenge in the management of PCa is discriminating between the 
indolent and aggressive disease, and the early detection of PCa is important to guide treatment strategies. In this context, the prostate-specific antigen (PSA) test, as well as the tumor, node, metastasis (TNM) classification and the Gleason score in prostate biopsies, are routinely used for predicting the prognosis and informing treatment decisions [2]. The current treatments for PCa include radical prostatectomy, performed as a curative therapy, and androgen deprivation treatment, which is typically achieved using androgen receptor antagonists.

Solid tumors employ aerobic glycolysis to generate energy (the so-called Warburg effect). To support transformation, cancer cells can rewire their metabolism to sustain the production of energy, and, in this sense, fatty acid metabolism has recently received particular attention. Cancer cells can fuel their fatty acid metabolism through the activation of pathways such as the PI3K-AKT-mTORC1 signaling axis [3]. Fatty acids can be obtained from de novo lipogenesis and from exogenous uptake, which are facilitated by transporters such as CD36 (cluster of differentiation 36) and FABP (fatty acid-binding protein). Fatty acids can be stored as lipid droplets and used for acetyl-CoA production through $\beta$-oxidation. De novo lipogenesis relies on the enzymatic activities of ACLY (ATP citrate synthase), ACACA (acetyl-CoA carboxylase alpha) and FASN (fatty acid synthase), which generate diverse lipid species [3,4].

Aberrancies in cellular metabolism in PCa are recognized hallmarks of malignant transformation [5]. Indeed, the large amount of citrate that would normally be secreted functions as an intermediate in the citric acid cycle and as a substrate for de novo fatty acid synthesis. In this regard, the dysregulation of lipid metabolism involving the upregulation of lipogenic enzymes and of enzymes that function to oxidize fatty acids as an energy source is a common finding in PCa [6]. Furthermore, a recent systematic review and meta-analysis found that patients with PCa had higher fasting serum insulin and higher homeostasis model assessment-estimated insulin resistance (HOMA-IR) levels, especially those over 65 years of age [7]. Insulin is a known growth factor for PCa cells [8]; however, its role in PCa etiology remains unclear.

A strong association is known to exist between chronic inflammation and several types of cancer, and inflammatory cytokines have been considered as potential mediators of prostate carcinogenesis [9]. Yet, the modulatory actions of the inflammatory factors on energy metabolism in prostate epithelial cells have been poorly investigated.

The tumor necrosis factor-like weak inducer of apoptosis (TWEAK) is a cytokine expressed mainly by monocytes and macrophages [10]. TWEAK can exist as a full-length, membrane-anchored form (mTWEAK) on the cell surface [10] or as a biologically active soluble form (sTWEAK) generated by the proteolytic processing of mTWEAK, which is detectable in the extracellular milieu and biofluids [11]. Both forms functionally engage the fibroblast growth factor-inducible 14 (Fn14) receptor to activate downstream signaling pathways [12,13]. Early studies on the TWEAK/Fn14 axis in PCa identified Fn14 as a candidate for enhanced PCa growth in xenografted mice under high-fat feeding [14] and with a probable role in invasion, migration and proliferation in PCa cell models [15]. Similarly, the overexpression of Fn14 contributes to multiple malignant cellular phenotypes associated with cancer progression in androgen-independent PCa cell lines, in part controlled by the tissue remodeling enzyme matrix metalloproteinase 9 (MMP-9) [15]. The finding of a potential relationship between the Fn14 expression and patient outcome in patients undergoing radical prostatectomy for localized PCa [15] points to Fn14 as a novel prognostic biomarker candidate in PCa. In an effort to identify new PCa biomarkers in liquid biopsies, we recently established that semen levels of sTWEAK were lower in high-risk patients than in low-risk peers and that this reduction was accompanied by a trend for an increase in the Fn14 mRNA expression levels in seminal cell sediment [16]. More recently, we found that sTWEAK semen levels and TWEAK-induced oncogenic shuttle microRNAs could be integrated into an improved PCa prognostic panel based only on information obtained from semen [17]. 
sTWEAK serum levels are significantly and independently associated with an increased risk of type 2 diabetes and metabolic syndrome [18], collectively highlighting a possible role for STWEAK in the pathogenesis of metabolic diseases. This is supported by the finding that sTWEAK can modulate in vitro human hepatocyte lipid accumulation [19].

Given the established relationship between sTWEAK and metabolic-related pathologies, we sought to study whether there was a possible link between circulating sTWEAK levels and the metabolic status of patients with PCa. To this end, we investigated sTWEAK serum levels in a well-characterized cohort of patients with PCa in relation to glucose and lipid metabolic profiles. Additionally, to better understand the impact of inflammation on metabolic dysregulation in PCa, we used cell models to investigate whether this cytokine, previously reported to be present in the prostate microenvironment, could alter local glucose and/or lipid metabolism in PCa.

\section{Materials and Methods}

\subsection{Study Population}

This was a cross-sectional, case-control, retrospective study of 76 patients with PCa and 159 controls. Control patients with PSA levels below average for their age $(<1.3 \mathrm{ng} / \mathrm{mL}$ PSA with ages 60-69 years; $<1.7 \mathrm{ng} / \mathrm{mL}$ PSA with ages 70-79 years) [20] were recruited from outpatients in the hospital Joan XXIII, Tarragona, Spain. Patients with PCa, recruited from the same hospital, had undergone radical prostatectomy, which was supported by the European guidelines and was a treatment option in our Urology Department. The surgeries were performed between 2015 and 2020. The grades, groups and stages of the tumors were determined according to the 2014 International Society of Urological Pathology (ISUP) Gleason Grading (GG) and TNM classification. The patients were stratified into two groups, according to the ISUP GG: low-risk (ISUP Groups I and II) and high-risk (ISUP Groups III, IV and V). This study was performed according to the provisions of the Declaration of Helsinki and was approved by our local ethics committee and adhered to the current legal regulations (Biomedical Research Law 14/2007, Royal Decree of Biobanks 1716/2011 and Organic Law 15/1999 of September 13 Protection of Personal Data). All methods were approved and performed in accordance with the guidelines and regulations of the Ethical Committee for Clinical Research (CEIM) from the Pere Virgili Research Institute (Ref. CEIM171/2017; CEIM205/2020 approved on 02/10/2017 and 24/09/2020, respectively).

\subsection{Biochemical Data}

All patients underwent anthropometric measurements. Blood was extracted for measurements of plasma lipids, glucose, insulin and sTWEAK after an 8-h fast. The glucose and lipid levels were determined by standard laboratory methods. Serum insulin was measured in duplicate using a monoclonal immunoradiometric assay (Medgenix Diagnostics, Fleunes, Belgium). The serum $\gamma$-glutamyltransferase (GGT), uric acid and creatinine levels were determined using enzymatic methods. The HOMA-IR index was calculated using the following formula:

$$
\text { serum glucose }(\mathrm{mmol} / \mathrm{L}) \times \text { serum insulin }(\mathrm{mU} / \mathrm{L}) / 22.5 \text {. }
$$

Serum concentrations of sTWEAK were determined in duplicate by the enzyme-linked immunosorbent assay using the human TWEAK/TNFSF12 Kit \#DY1090 (R\&D Systems Europe, Abingdon, Oxon, UK). The intra- and inter-assay coefficients of variation were $2.5 \%$ and $7.0 \%$, respectively, and the sensitivity of the test was $62 \mathrm{pg} / \mathrm{mL}$.

\subsection{Cell Culture and Treatments}

The prostate cancer cell lines PC-3 (androgen-insensitive) and LNCaP (androgensensitive) and the histologically normal prostate epithelial RWPE-1 cell line (immortalized with papilloma virus 18) were purchased from Sigma-Aldrich (Barcelona, Spain). PC-3 cells were cultured in Ham's F-12K (Kaighn's) Medium (1:1 mixture) with L-glutamate (Invitrogen/Gibco, Fisher Scientific SL, Madrid, Spain). LNCaP cells were cultured in RPMI 
1640 medium (Merck KGaA, Darmstadt, Germany) supplemented with 1-mM sodium pyruvate (Gibco). PC-3 and LNCaP cultures were supplemented with $10 \%$ fetal bovine serum. RWPE-1 cells were cultured in keratinocyte serum-free medium plus $5 \mu \mathrm{g} / \mathrm{mL}$ bovine pituitary extract. All the cells were supplemented with $1 \times$ antibiotic-antimycotic (Gibco) and $5 \mu \mathrm{g} / \mathrm{mL}$ Plasmocin ${ }^{\mathrm{TM}}$ (InvivoGen, San Diego, CA, USA). Where indicated, the cells were grown in serum-deprived medium overnight before stimulation for 24 or $48 \mathrm{~h}$ with $100 \mathrm{ng} / \mathrm{mL}$ human recombinant TWEAK (PeproTech, bioNova cientifica, Barcelona, Spain) and were cultured in a humidified $5 \% \mathrm{CO}_{2}$ atmosphere at $37^{\circ} \mathrm{C}$.

\section{4. siRNA Experiments}

An siRNA targeting the human Fn14 gene (TNFRSF12A siRNA sequence sense $5^{\prime}$ GAGGGAGAAUUUAUUAAUATT- $3^{\prime}$ and antisense $5^{\prime}$-UAUUAAUAAAUUCUCCCUC-3') and an siRNA control (MOCK) were purchased from Thermo Fisher Scientific (Invitrogen, Madrid, Spain). The transfected siRNAs concentration used was $50 \mathrm{nM}$, as recommended by the manufacturer, and has been tested elsewhere [21]. Opti-MEM and Lipofectamine 3000 were used for the transfection (Thermo Fisher Scientific). Twenty-four hours after the transfection, the cells were stimulated or not with $100 \mathrm{ng} / \mathrm{mL}$ human recombinant TWEAK for $48 \mathrm{~h}$ and then collected for gene expression and protein analysis.

\subsection{Inhibitor Treatments}

The MEK inhibitor PD184352 (Sigma-Aldrich, St. Louis, MO, USA) was used at a final concentration of $10 \mu \mathrm{M}$, which led to successful p-ERK inhibition [22]. The AKT inhibitor MK2206 (Deltaclon S.L., Madrid, Spain) was used at a final concentration of $1 \mu \mathrm{M}$ [23]. Parthenolide (Sigma-Aldrich) and Amgen16 (Sigma-Aldrich) were used for NF-kB canonical and noncanonical inhibition, respectively, at $10 \mu \mathrm{M}$ [24,25]. PC-3 cells were treated with inhibitors for $1 \mathrm{~h}$ before stimulation with $100 \mathrm{ng} / \mathrm{mL}$ TWEAK (or vehicle) for $24 \mathrm{~h}$. RNA was then extracted for gene expression analysis.

\subsection{Determination of Fatty Acid Uptake and Lipid Accumulation}

PC-3 cells were seeded in 96-well clear flat-bottom black plates, and treatments with sTWEAK and with siFn14 were performed as described above. To quantify the fatty acid uptake, the growth medium was exchanged with TF2-C12 fatty acid (Sigma-Aldrich), and the cells were incubated at $37^{\circ} \mathrm{C}$ for $30 \mathrm{~min}$. The cellular uptake was measured on a Varioskan Lux Reader (Thermo Fisher Scientific) by the measurement of fluorescence intensity $(\lambda$ ex $=485 / \lambda$ em $=515 \mathrm{~nm})$. The lipid content was measured with Nile Red (Sigma-Aldrich). Briefly, the media were removed, cells were washed twice with PBS and lipids were stained with $1.1 \mathrm{mg} / \mathrm{mL}$ Nile Red for $15 \mathrm{~min}$ at $37^{\circ} \mathrm{C}$. After incubation, the cells were washed with PBS, followed by measurement of the fluorescence intensity $(\lambda$ ex $=488 / \lambda$ em $=590 \mathrm{~nm})$.

\subsection{Gene Expression Analysis}

RNA was extracted, cDNA was synthesized, and real-time quantitative PCR was performed on a 7900HT Fast Real-Time PCR system (Applied Biosystems, Foster City, CA, USA), as described [26]. Gene quantification was performed using the following commercial Taqman assays (Applied Biosystems): ACACA, ACLY, FASN, CPT1, PNPLA2, SREBP-1, PPARG, FABP4, CD36, SLC2A1, SLC2A4, HK1, PFKM, PDK4, PDHA1 and PKM2. The cycle threshold (CT) value for each sample was normalized to the expression of PPIA (peptidylprolyl isomerase A), used as an internal control. SDS software (2.3, Applied Biosystems, Foster City, California, USA) and RQ Manager 1.2 (Applied Biosystems) were used to analyze the results with the comparative CT method $\left(2^{-\Delta \Delta C T}\right)$.

\subsection{Western Blot Analysis}

A total of $25 \mu \mathrm{g}$ of protein was fractionated on $4-15 \%$ gradient SDS-PAGE gels and transferred to nitrocellulose membranes using the standard protocols. The following 
primary antibodies were used: p-ERK1/2 (\#4370), p-p38 (\#4511), p-JNK (\#4668), p-IKB (\#2859), NF-kB2 p100/p52 (\#4882), Fn14 (\#4403), p-AktS473 (\#9271), FASN (\#3180S) and CPT-1A (\#12252) and were purchased from Cell Signaling Technology (Danvers, MA, USA), FABP4 (SC18661) was purchased from Santa Cruz Biotechnology (Dallas, TX, USA) and anti- $\$$-actin was purchased from Sigma-Aldrich. The antibodies were used at the dilutions recommended by the manufacturers.

\subsection{Statistical Analysis}

For the clinical, anthropometrical and analytical parameters, all data were tested for normality using the Shapiro-Wilk test. Normally distributed data were reported as the mean and standard deviation (SD). Differences between patient groups were tested with the Mann-Whitney $U$ test. Spearman's correlation coefficients were used to analyze the relationship between normally and non-normally distributed parameters. Multiple logistic regression analyses were employed (stepwise backward selection procedures) to determine whether the STWEAK levels were associated with the presence of PCa. In vitro experimental results were presented as the mean and standard error of the mean (SEM) of 3 to 4 independent experiments. The differences were tested with the unpaired two-tailed Student's $t$-test. The statistical software SPSS 21.0 (IBM, Madrid, Spain) and R package (R Core Team (2013). R: A language and environment for statistical computing. R Foundation for Statistical Computing, Vienna, Austria) were used for the analyses. Significance was considered at a $p$-value $<0.05$.

\section{Results}

\subsection{Low Concentrations of sTWEAK Are Associated with $\mathrm{PCa}$}

The baseline characteristics of the 76 patients with PCa and the 159 controls included in the present study are shown in Table 1. Fasting plasma glucose, serum insulin, HOMAIR, HDL-cholesterol, creatinine and total serum PSA levels were all significantly higher in patients with PCa than in the controls (Table 1), whereas the opposite was seen for the serum sTWEAK levels (Figure 1A). Spearman's correlation coefficients are described in Figure 1B. The most relevant associations were the negative associations between the sTWEAK and serum PSA $(r=-0.178, p=0.006)$, insulin $(r=-0.156, p=0.017)$ and HOMA-IR $(r=-0.164 ; p=0.012)$ and the positive associations with the total cholesterol $(r=0.234 ; p<0.001)$ and LDL cholesterol $(r=0.163, p=0.012)$. Additionally, the serum PSA levels were positively associated with fasting glucose $(r=0.194, p=0.003)$, insulin $(r=0.473, p<0.001)$, HOMA-IR $(r=0.465, p<0.001)$ levels and HDL cholesterol $(r=0.149$, $p=0.022)$ and negatively associated with serum creatinine $(r=-0.172, p=0.008)$.

Table 1. Anthropometric and analytical characteristics of the studied cohorts.

\begin{tabular}{|c|c|c|c|}
\hline Number of Patients & $\begin{array}{c}\text { Controls } \\
n=159 \\
\text { Mean } \pm \text { SD }\end{array}$ & $\begin{array}{c}\text { PCa Patients } \\
n=76 \\
\text { Mean } \pm \text { SD }\end{array}$ & $p$-Value \\
\hline \multicolumn{4}{|c|}{ Anthropometric Parameters } \\
\hline Age (years) & $63.54 \pm 7.26$ & $63.51 \pm 6.66$ & 0.953 \\
\hline $\mathrm{BMI}\left(\mathrm{kg} / \mathrm{m}^{2}\right)$ & $28.60 \pm 2.31$ & $28.24 \pm 3.97$ & 0.067 \\
\hline ISUP GG $(n / \%)$ & - & - & - \\
\hline Low Risk (I and II) & - & $48(63 \%)$ & - \\
\hline $\begin{array}{l}\text { High Risk (Group III, IV, V) } \\
\text { Glycemic profile }\end{array}$ & - & $28(37 \%)$ & - \\
\hline
\end{tabular}


Table 1. Cont.

\begin{tabular}{|c|c|c|c|}
\hline Number of Patients & $\begin{array}{c}\text { Controls } \\
n=159 \\
\text { Mean } \pm \text { SD }\end{array}$ & $\begin{array}{c}\text { PCa Patients } \\
n=76 \\
\text { Mean } \pm \text { SD }\end{array}$ & $p$-Value \\
\hline \multicolumn{4}{|c|}{ Anthropometric Parameters } \\
\hline Glucose (mmol/L) & $5.20 \pm 0.53$ & $6.03 \pm 1.35$ & $<0.001$ \\
\hline Insulin (pmol/L) & $30.60 \pm 19.93$ & $94.53 \pm 55.33$ & $<0.001$ \\
\hline HOMA-IR & $1.04 \pm 0.74$ & $3.75 \pm 2.63$ & $<0.001$ \\
\hline Diabetes $(n / \%)$ & $0(0 \%)$ & $8(10.6 \%)$ & $<0.001$ \\
\hline \multicolumn{4}{|l|}{ Lipid profile } \\
\hline Cholesterol (mmol/L) & $5.17 \pm 0.99$ & $4.96 \pm 1.04$ & 0.233 \\
\hline HDL cholesterol (mmol/L) & $1.22 \pm 0.28$ & $1.47 \pm 0.66$ & 0.001 \\
\hline LDL cholesterol (mmol/L) & $2.87 \pm 0.71$ & $3.07 \pm 1.23$ & 0.185 \\
\hline Triglycerides (mmol/L) & $1.50 \pm 0.90$ & $1.49 \pm 0.81$ & 0.925 \\
\hline \multicolumn{4}{|l|}{ Hepatic profile } \\
\hline GGT ( $\mu$ kat/L) & $0.64 \pm 0.67$ & $0.70 \pm 0.79$ & 0.404 \\
\hline \multicolumn{4}{|l|}{ Renal profile } \\
\hline Uric acid ( $\mu \mathrm{mol} / \mathrm{L})$ & $366.46 \pm 78.47$ & $413.27 \pm 379.57$ & 0.527 \\
\hline Creatinine $(\mu \mathrm{mol} / \mathrm{L})$ & $76.74 \pm 13.31$ & $70.98 \pm 14.21$ & $<0.001$ \\
\hline Total PSA $(\mu \mathrm{g} / \mathrm{L})$ & $1.64 \pm 2.14$ & $10.06 \pm 7.73$ & $<0.001$ \\
\hline sTWEAK & $1583.13 \pm 1348.60$ & $1197.20 \pm 579.57$ & $<0.001$ \\
\hline
\end{tabular}

Abbreviations: PCa, Prostate Cancer; SD, Standard deviation; ISUP (GG): International Society of Urological Pathology Gleason Grading; BMI, Body mass index; cc, centiliters; HOMA-IR, Homeostatic Model Assessment for Insulin Resistance; HDL, High-density lipoprotein; LDL, Low-density lipoprotein; GGT, Gamma Glutamyltransferase; PSA, Prostate-specific antigen; sTWEAK, soluble tumor necrosis factor-like weak inducer of apoptosis.

A multivariate ordered logistic regression analysis was used to evaluate the independent predictors associated with PCa in the whole population. The variables included in the final model were the insulin, creatinine, HDL cholesterol, glucose, HOMA-IR, sTWEAK and PSA levels. The results showed that HOMA-IR (odds ratio (OR) $=0.126, p<0.001$ ), PSA serum levels $(\mathrm{OR}=0.539, p<0.001)$, HDL cholesterol $(\mathrm{OR}=0.040 p<0.001)$ and lower sTWEAK serum levels $(\mathrm{OR}=1.002 p<0.001)$ were independently associated with the presence of PCa (Figure 1C).

\section{2. sTWEAK Treatment of PCa Cell Lines Alters the Expression of Genes Related to Lipid Metabolism but Not to Glucose Metabolism}

To assess whether sTWEAK influences PCa cell metabolism, we examined for possible changes elicited by sTWEAK on the expression of the selected genes associated with glucose metabolism in two PCa cell lines (LNCaP and PC-3 cells) and a control, epithelial cell line (RWPE-1). All cell types expressed the gene for Fn14, which was upregulated under sTWEAK stimulation (Figure S1). The cell cultures were treated with sTWEAK for 24 and $48 \mathrm{~h}$. The results showed that the expression of SLC2A1 and SLC2A4 (glucose transporters 1 and 4) decreased significantly in RWPE-1 cells after treatment with sTWEAK (48 h) but not in PC-3 and LNCaP cells (Figure 2A). Gene activity in the "preparatory phase" (energy consuming) of glycolysis was lower in PC-3 and RWPE-1 cells after a 24- and 48-h sTWEAK treatment, as shown by a significant decrease in the expression of hexokinase 1 (HK1) and 6-phosphofructo-1-kinase (PFKM), whereas no significant changes were observed in LNCaP cells (Figure 2B). 
A)
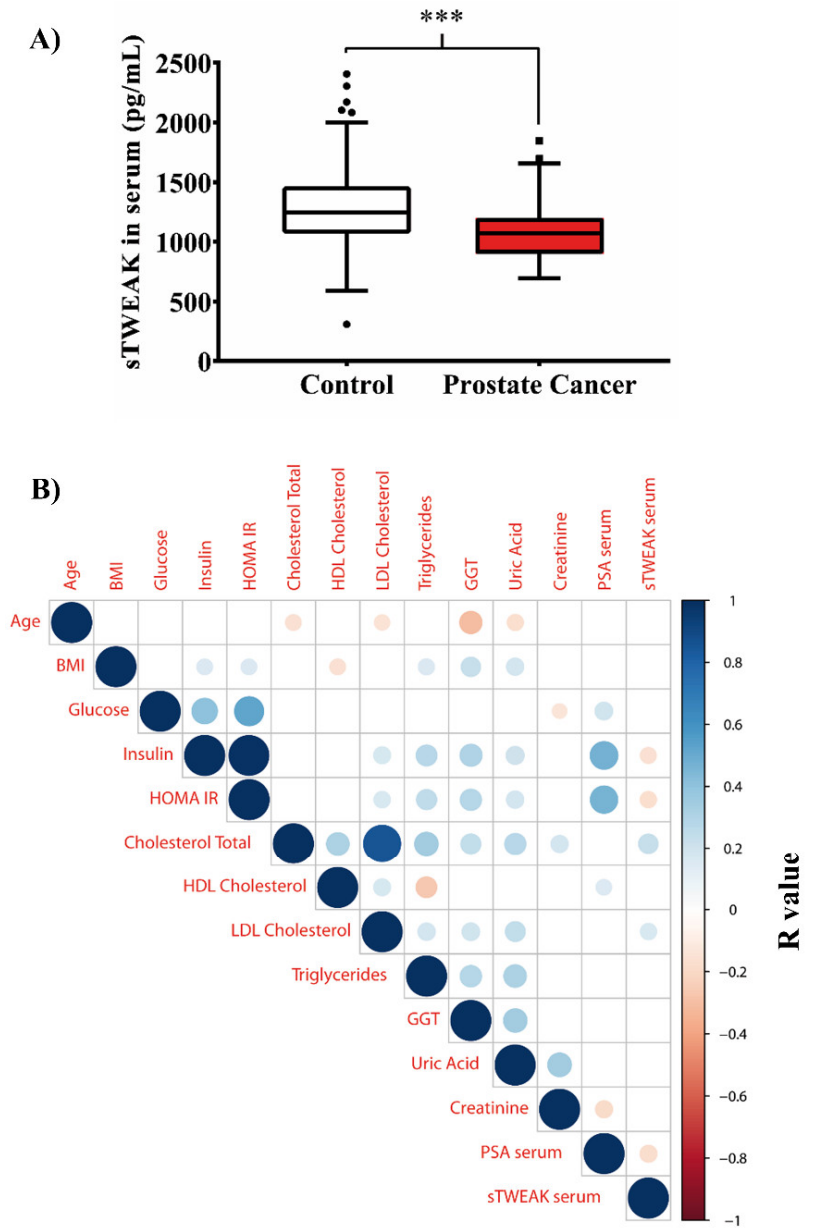

C)

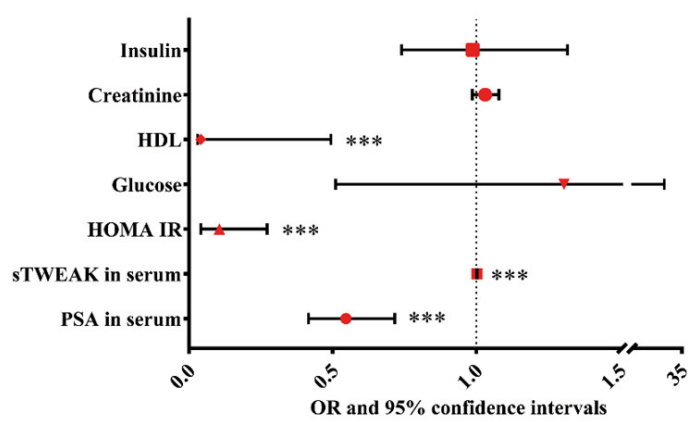

Figure 1. Circulating serum levels of sTWEAK in PCa. (A) Box plot (each box shows the median, quartiles and extreme values) representation of the sTWEAK serum levels in the control and PCa cohorts. ${ }^{* * *} p<0.001$. (B) Spearman's correlation matrix. Positive correlations are displayed in graded blue colors and negative correlations in graded red colors. Correlations with $p$-values $\geq 0.05$ are considered as insignificant and are left blank. The color intensity and the size of the circle are proportional to the correlation coefficients. On the right side of the correlogram, the color legend shows the correlation coefficients and the corresponding colors. (C) Relationship between PCa and clinical, anthropometric and analytical parameters. Odds ratios (OR values in different red shapes for each parameter) and 95\% confidence intervals. Dotted horizontal line: OR greater than 1 indicates that variable is more likely to occur in PCa and, an OR less than 1 indicates that variable is less likely to occur in PCa. ${ }^{* * *} p<0.001$. Abbreviations: BMI, body mass index; HOMA-IR, homeostatic model assessment for insulin resistance; HDL, high-density lipoprotein; LDL, low-density lipoprotein; GGT, gamma glutamyltransferase; PSA, prostate-specific antigen; sTWEAK, soluble tumor necrosis factor-like weak inducer of apoptosis. 
(A)

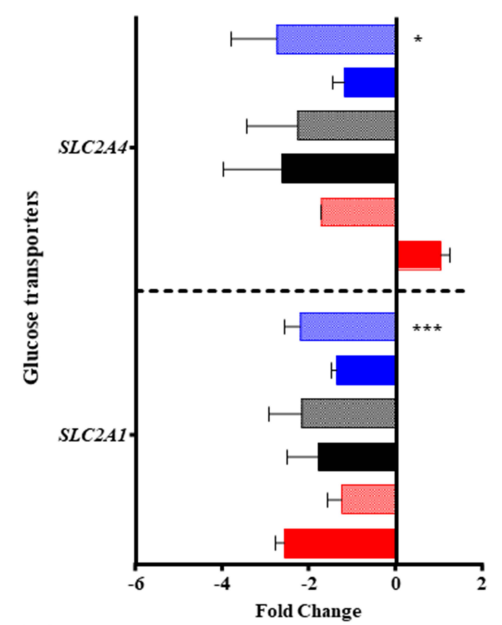

(D)

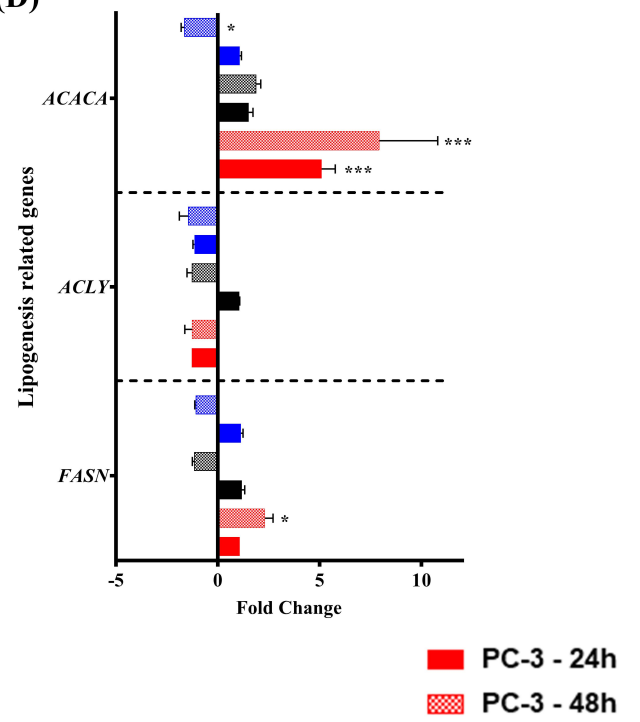

(B)

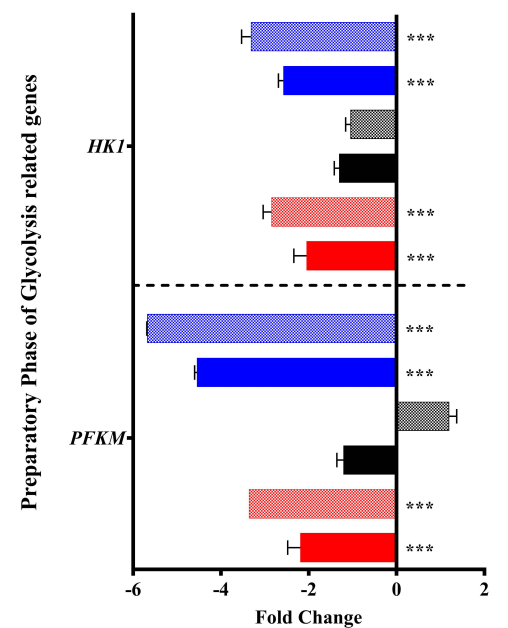

(E)

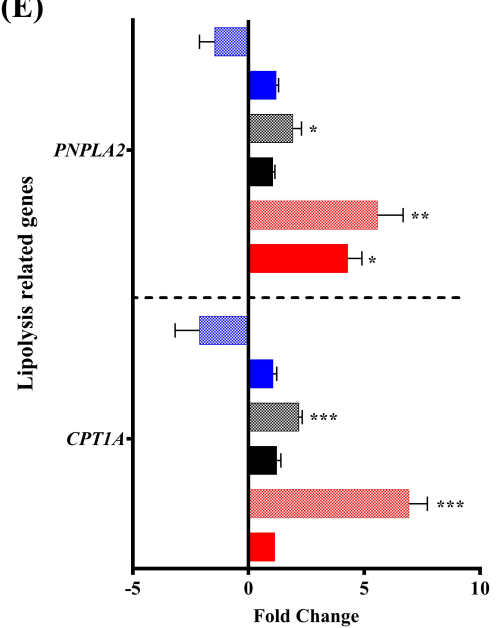

(F)
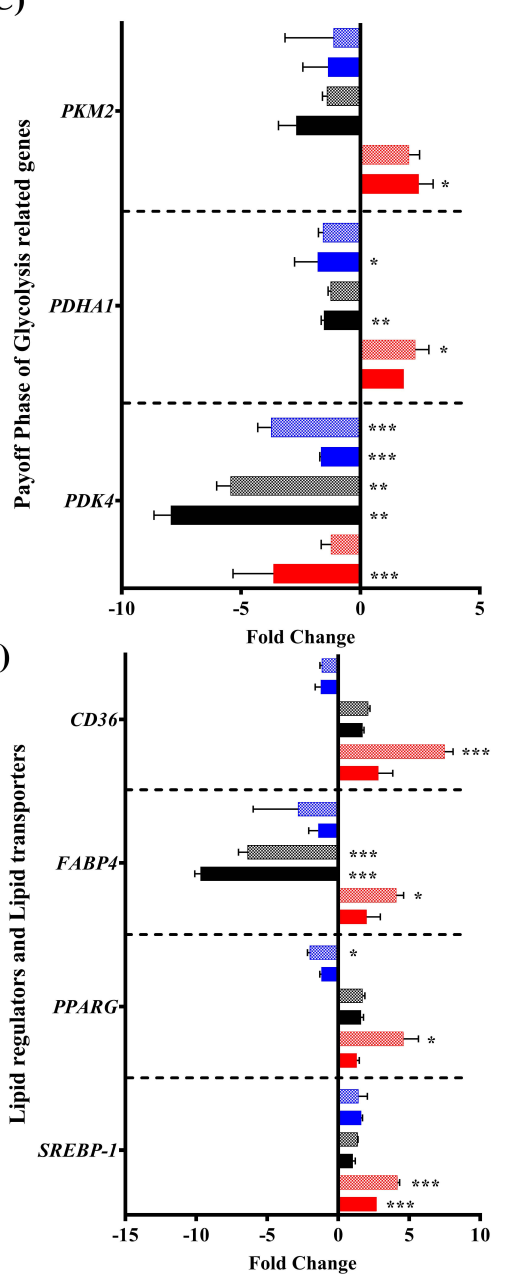

RWPE-1 - 24h

RWPE-1 - 48h

Figure 2. Effect of exogenous sTWEAK on the expression of the genes related to glucose and lipid metabolism. The glucose and lipid metabolism-related genes were evaluated by RT-qPCR after treating PC-3, LNCaP and RWPE-1 cells with $100 \mathrm{ng} / \mathrm{mL}$ TWEAK for 24 or $48 \mathrm{~h}$. (A) Glucose transporters. (B) Preparatory phase of glycolysis. (C) Payoff phase of glycolysis. (D) Lipogenesis-related genes. (E) Lipolysis-related genes. (F) Lipid regulators and lipid transporter genes. Results are presented as the mean \pm SEM of 4 independent experiments of the fold change expression with respect to the nontreated respective controls. ${ }^{*} p<0.05,{ }^{* *} p<0.01$ and ${ }^{* * *} p<0.001$. Abbreviations: SLC2A1 and SLC2A4, glucose transporter 1 and 4, respectively; HK1, hexokinase 1; PFKM, 6-phosphofructo-1-kinase; PKM2, pyruvate kinase M2; PDHA1, pyruvate dehydrogenase alpha 1; $P D K 4$, pyruvate dehydrogenase kinase 4; $A C A C A$, acetyl-CoA carboxylase alpha; $A C L Y$, ATP-citrate synthase; FASN, fatty acid synthase; PNPLA2, patatin-like phospholipase domain containing 2; CPT1A, carnitine palmitoyltransferase 1A; CD36, CD36 molecule; FABP4, fatty acid-binding protein 4; PPARG, peroxisome proliferatoractivated receptor gamma; $S R E B P-1$, sterol regulatory element-binding transcription factor 1; PC-3, androgen-independent human advanced adenocarcinoma prostate cancer cell line, LNCaP, androgen-sensitive human prostate adenocarcinoma cell line; RWPE-1 epithelial cell line derived from the peripheral zone of a histologically normal adult human prostate.

We also examined the "payoff phase" (energy production) of glycolysis, which showed a differential response to sTWEAK in the PCa cell lines studied. Pyruvate kinase M2 (PKM2) gene expression was unchanged in LNCaP and control RWPE-1 cells, whereas pyruvate dehydrogenase alpha 1 (PDHA1) gene expression was significantly decreased at $24 \mathrm{~h}$. By contrast, the gene expression of PKM2 and PDHA1 significantly increased at 24 and $48 \mathrm{~h}$, respectively, in PC-3 cells. Additionally, the expression of pyruvate dehydrogenase kinase 
4 (PDK4) was significantly lower in LNCaP cells at $24 \mathrm{~h}$ and in RWPE- 1 cells at 24 and $48 \mathrm{~h}$ after TWEAK treatment (Figure 2C).

The downregulation of PDK4 and concomitant upregulation of PDHA1 in PC-3 cells is noteworthy, as $P D K 4$ is an inhibitor of $P D H A 1$, which might indicate that the pyruvate generated by PKM2 is converted into acetyl-CoA by the action of PDHA1, thus entering the citric acid cycle and fueling lipogenesis in PC-3 cells.

Next, we investigated whether STWEAK administration altered the expression of the genes involved in lipid metabolism in PCa cell lines. We found that sTWEAK treatment significantly increased the expression levels of lipogenesis-related genes such as acetylCoA carboxylase alpha $(A C A C A)$ at 24 and $48 \mathrm{~h}$ and fatty acid synthase (FASN) at $48 \mathrm{~h}$ of stimulation but only in PC-3 cells (Figure 2D). By contrast, the mRNA levels of $A C A C A$ were significantly lower under sTWEAK stimulation in the control RWPE-1 after $48 \mathrm{~h}$. No changes were observed in the ACACA expression in LNCaP cells. Additionally, no changes were observed for the ATP citrate synthase gene expression (ACLY) in any cell model.

Examination of the lipolysis-related genes patatin-like phospholipase domain containing 2 (PNPLA2) and carnitine palmitoyltransferase $1 \mathrm{~A}(C P T 1 A)$ revealed that both were significantly upregulated in PC-3 and LNCaP cells $48 \mathrm{~h}$ after sTWEAK administration, but no changes were observed in RWPE-1 cells (Figure 2E).

Other genes involved in lipid metabolism regulation, such as peroxisome proliferatoractivated receptor gamma (PPARG) and sterol regulatory element binding transcription factor 1 (SREBP-1), were exclusively and significantly upregulated by sTWEAK treatment in PC-3 cells at $48 \mathrm{~h}$, whereas PPARG was significantly downregulated in RWPE-1 cells (Figure 2F). Regarding lipid transporter-related genes, we observed a significant increase in a cluster of differentiation 36 gene expression (CD36) in sTWEAK-treated PC-3 at 48 h, whereas fatty acid-binding protein 4 (FABP4) expression was significantly downregulated in LNCaP cells at 24 and $48 \mathrm{~h}$ but was upregulated in PC-3 cells at $48 \mathrm{~h}$ (Figure 2F). No sTWEAK-induced changes in lipid transporter-related genes were observed in RWPE-1 cells.

A correlation between gene and protein expression under sTWEAK stimulation was verified by Western blotting for the selected lipid metabolism-related genes (Figure S2 and its detailed information can be found in supplementary materials).

\subsection{Silencing of Fn14 (TNFRSF12A) Abrogates the Ability of sTWEAK to Modulate Lipid Metabolism Genes in PC-3 Cells}

Because lipid metabolism-related genes were significantly upregulated by sTWEAK in PC-3 cells, we chose this line for further analysis. To corroborate that the observed effects on the lipid metabolism were due to TWEAK/Fn14 engagement, we silenced the Fn14 gene (TNFRSF12A) using a specific siRNA. The cells were then stimulated with sTWEAK for $48 \mathrm{~h}$. Compared with MOCK-transfected cells, the siRNA-mediated knockdown of the Fn14 gene resulted in the complete reduction of its expression (mRNA and protein) (Figure 3A,B) after sTWEAK stimulation. Additionally, siFn14 treatment also abolished the significant sTWEAK-induced expression of genes involved in lipogenesis ( $A C A C A$ and FASN), lipolysis (PNPLA2 and CPT1A), lipid transport (CD36 and FABP4) and lipid regulation (PPARG and SREBP1) (Figure 3A), clearly indicating that the observed effects of sTWEAK were due to ligand-receptor binding. These effects were paralleled by a reduction in the protein expression of the selected proteins FASN, CPT1A and FABP4, as evaluated by Western blotting (Figure 3B). 
(A)

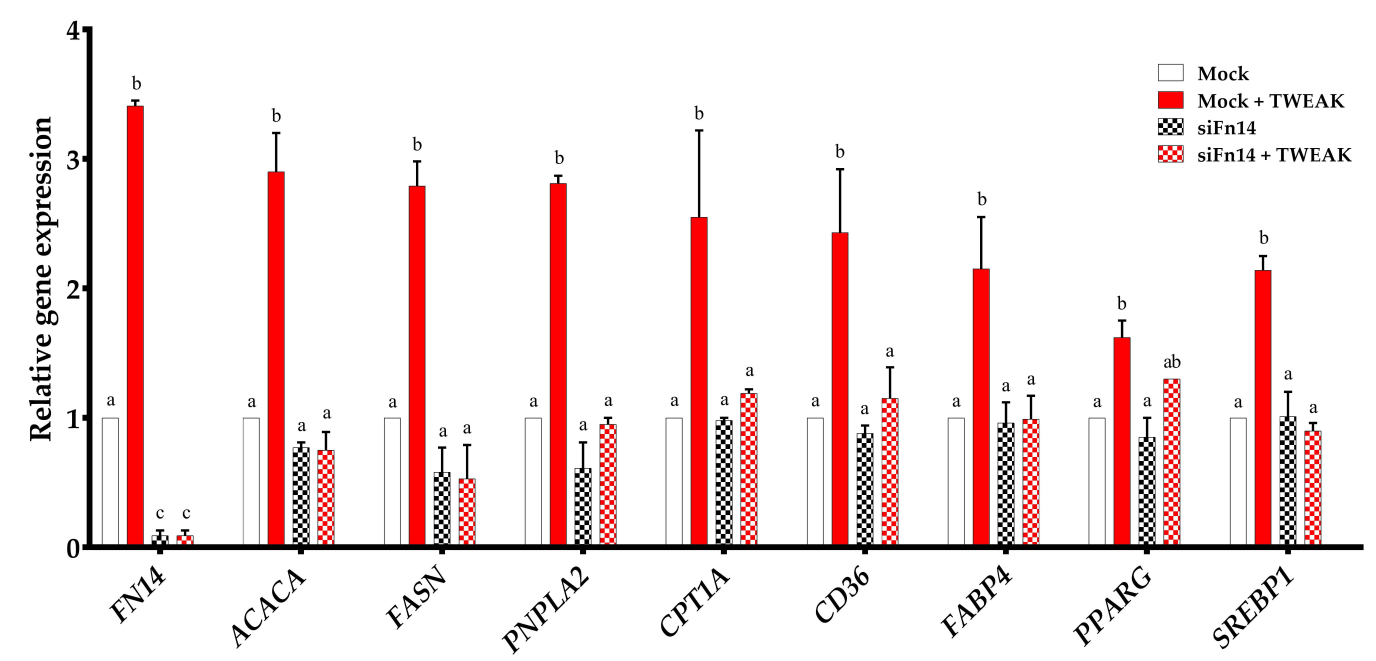

(B)
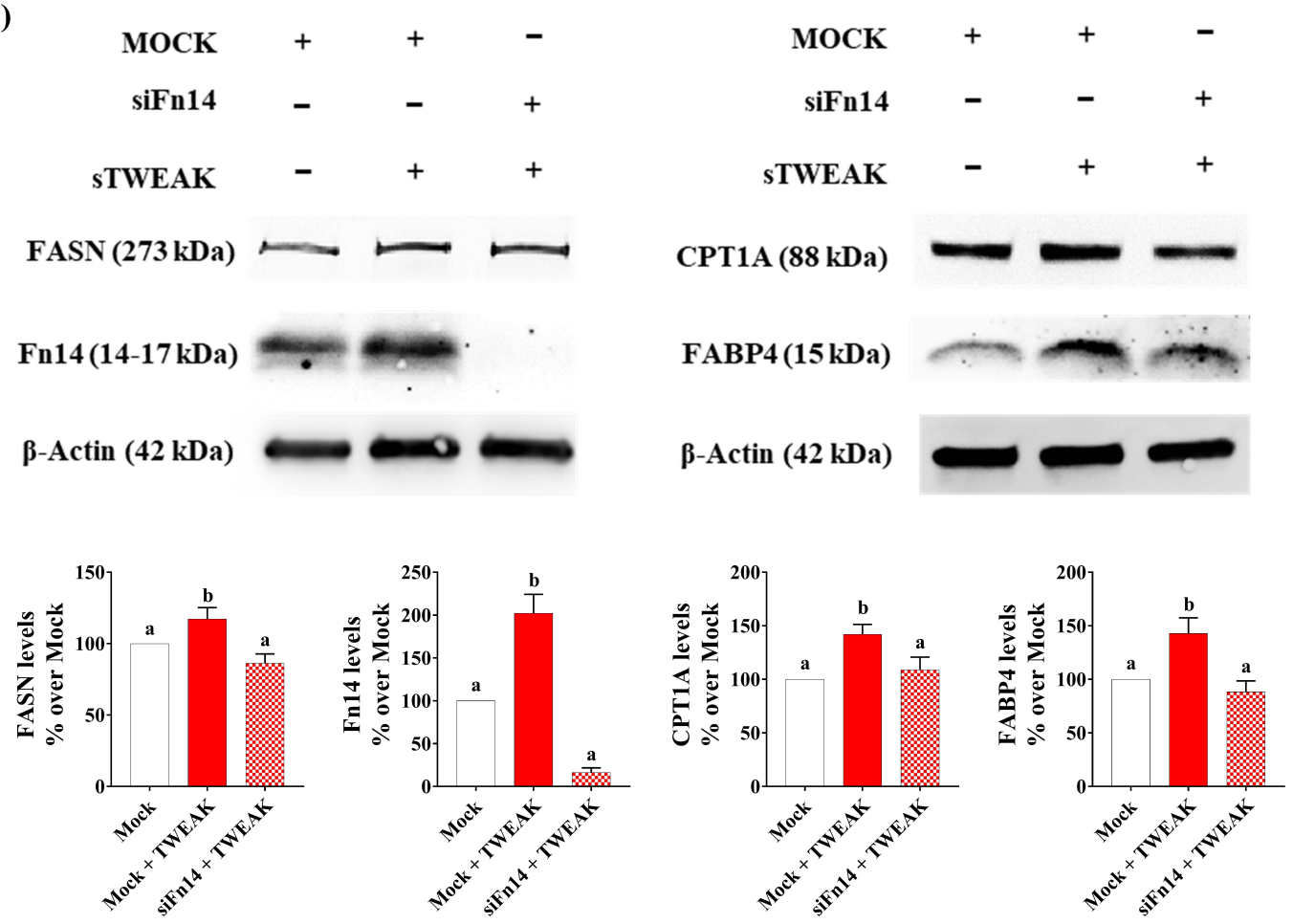

Figure 3. Inhibition of Fn14 in PC-3 cells blocks the sTWEAK-induced expression of lipid metabolism-related genes. (A) Fn14 was silenced in PC-3 cells using a Fn14 siRNA; a siRNA-negative sequence (MOCK) was used as a control. The cells were treated with $100 \mathrm{ng} / \mathrm{mL}$ TWEAK for $48 \mathrm{~h}$, and the lipid-related gene expression was measured by RT-qPCR. (B) Protein expression in Fn14 siRNA/MOCK-transfected PC-3 cells of FASN, CPT1, Fn14 and FABP4 by Western blotting. Detailed information about the Western blotting can be found at Figure S3. Different lettering over the boxes indicates statistical differences. Significant differences are established at $p<0.05$. Data are expressed as mean $\pm \operatorname{SEM}(n=3$ to 4 experiments). Abbreviations: Fn14, fibroblast growth factor-inducible 14; FASN, Fatty acid synthase; CPT1A, Carnitine palmitoyltransferase IA; siFn14, Fn14 small interfering RNA; FABP4, fatty acid-binding protein 4; ACACA, Acetyl-CoA Carboxylase Alpha; PNPLA2, patatin-like phospholipase 2; CD36, cluster of differentiation 36; PPARG, Peroxisome proliferator-activated receptor gamma; SREBP1, Sterol regulatory element-binding transcription factor 1; RT-qPCR, reverse transcription quantitative PCR. 


\section{4. sTWEAK Stimulates Fatty Acid Uptake and Lipid Accumulation in PC-3 Cells}

To directly measure the stimulatory effect of sTWEAK on the lipid metabolism, we utilized lipid uptake assays (Figure 4). As shown in Figure 4A, PC-3 cells treated for $48 \mathrm{~h}$ with sTWEAK significantly increased the fatty acid uptake, and this effect was significantly blocked-in cells pretreated with siFn14 before stimulation with sTWEAK. Notably, sTWEAK stimulation also increased lipid accumulation, as shown by Nile Red staining, Figure 4B).

(A)

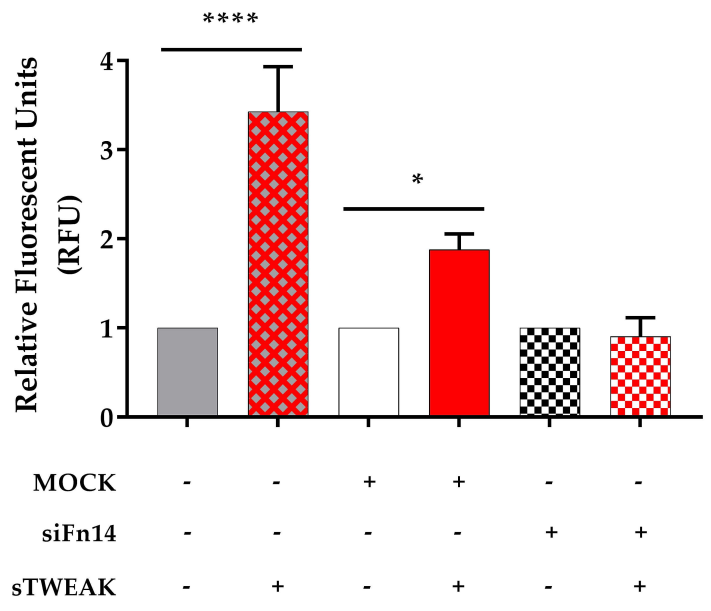

(B)

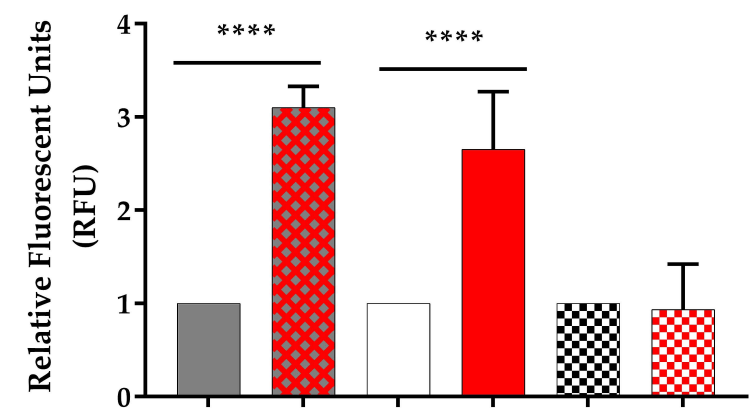

Control

Negative siRNA

TWEAK $100 \mathrm{ng} / \mathrm{ml}$
Nile Red

$\begin{array}{rcccccc}\text { MOCK } & - & - & + & + & - & - \\ \text { SiFn14 } & - & - & - & - & + & + \\ \text { STWEAK } & - & + & - & + & - & +\end{array}$

Negative siRNA + TWEAK $100 \mathrm{ng} / \mathrm{mL}$

Figure 4. sTWEAK stimulation induces lipid uptake and accumulation in PC-3 cells. Cells were treated with $100 \mathrm{ng} / \mathrm{mL}$ TWEAK for $48 \mathrm{~h}$. (A) Uptake of the fatty acid analog TF2-C12 was measured after $30 \mathrm{~min}$. (B) Lipid accumulation was measured with Nile Red dye after 15 min of incubation. Fn14 was silenced in PC-3 cells using a Fn14 siRNA; a siRNAnegative sequence (MOCK) was used as a control. Data are expressed as mean \pm SEM ( $n=4$ experiments). Significant differences: ${ }^{*} p<0.05$ and ${ }^{* * *} p<0.0001$. Abbreviations: sTWEAK, soluble TNF-like weak inducer of apoptosis; siFn14, Fn14 small interfering RNA.

\section{5. sTWEAK Stimulates Lipid Metabolism Genes in PC-3 Cells by Phosphorylating ERK1/2 and} AKT(ser473)

Activation of the phosphatidylinositol 3-kinase (PI3K-AKT-mTORC1) pathway in cancer cells reprograms the cellular metabolism by augmenting the activity of metabolic enzymes [3]. Mitogen-activated phosphokinase (MAPK) signaling pathways have also been implicated in redirecting energy utilization in malignant cells [27]. Examination of MAPK signaling in sTWEAK-stimulated PC-3 cells revealed the activation (phosphorylation) of ERK1/2, but no changes were observed for $\mathrm{p} 38$ or JNK1/2 phosphorylation (Figure $5 \mathrm{~A}$ ). We also observed that sTWEAK induced AKT (ser473) phosphorylation in PC-3 cells (Figure 5B). Similar results were observed for LNCaP cells, but AKT (ser473) phosphorylation occurred after a 6-h exposure to sTWEAK (Figure S4 and its detailed information can be found in supplementary materials). 
(A)
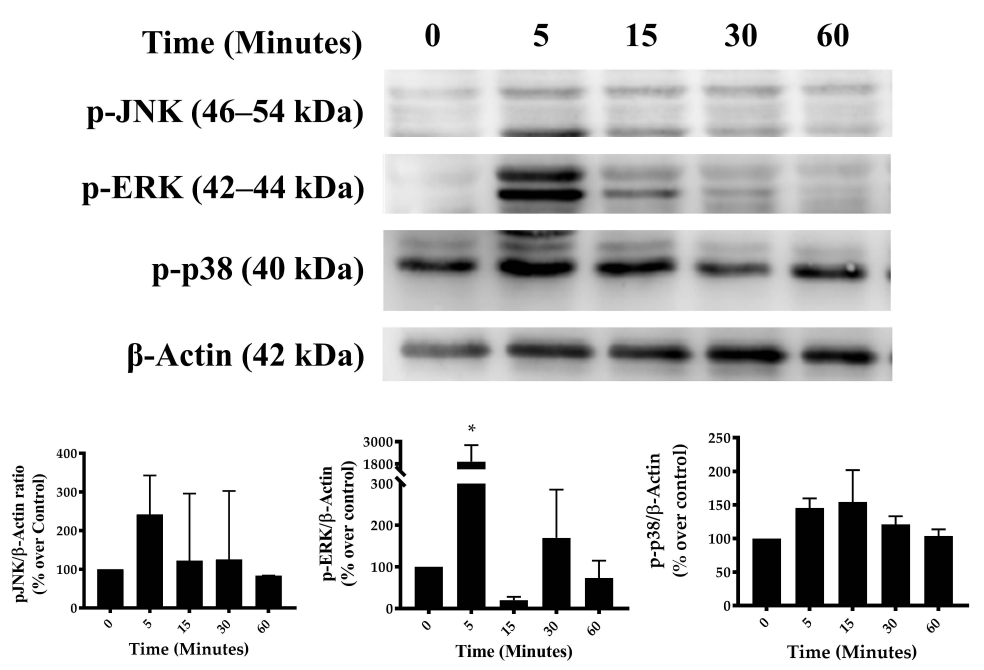

(B)

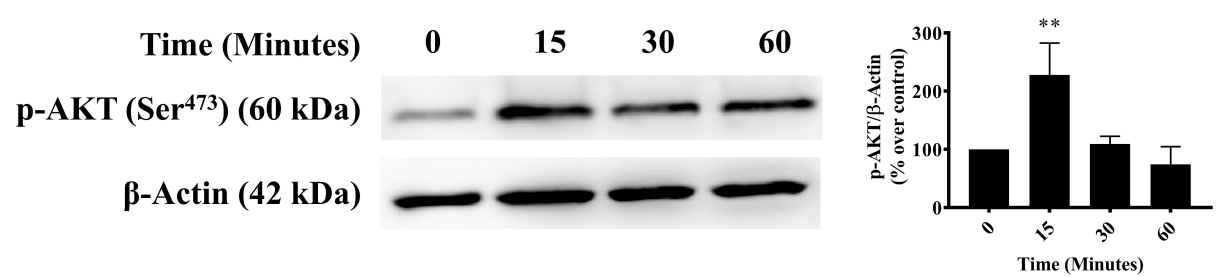

(C)

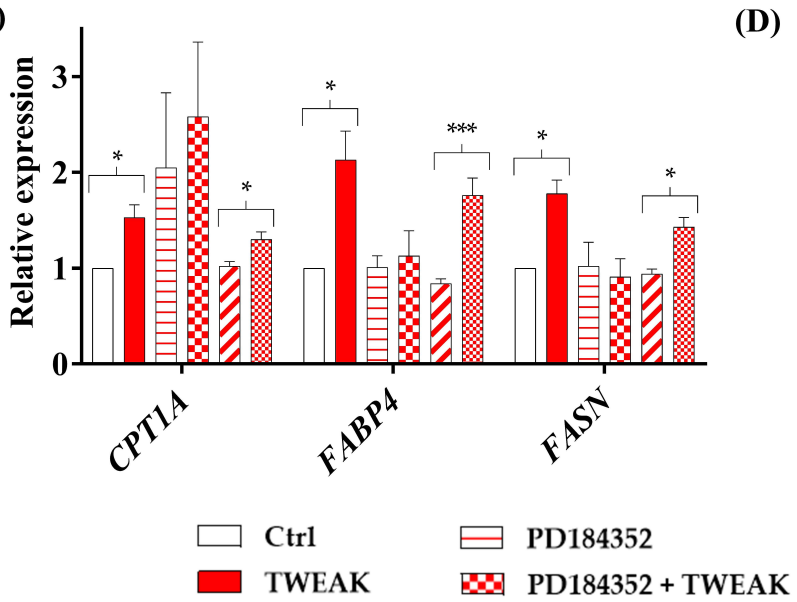

(D)

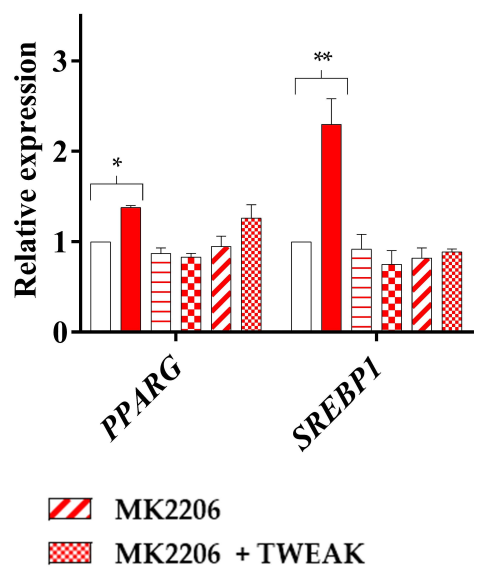

Figure 5. sTWEAK phosphorylates ERK1/2 and AKT(ser473) and regulates lipid-related gene expression in PC-3 cells. Cells were stimulated with $100 \mathrm{ng} / \mathrm{mL}$ sTWEAK at different time points. (A) MAPK and (B) AKT (ser473) phosphorylation were analyzed by Western blotting. Representative Western blots and a densitometry analysis of 3 independent experiments (mean \pm SEM) are shown. ${ }^{*} p<0.05$ and ${ }^{* *} p<0.01$. PC-3 cells were treated with $10 \mu \mathrm{M}$ ERK1/2 inhibitor (PD184352) or $1 \mu \mathrm{M}$ AKT inhibitor (MK2206) and then stimulated with $100 \mathrm{ng} / \mathrm{mL}$ sTWEAK for $24 \mathrm{~h}$. The gene expression of the (C) CPT1A, FABP4 and FASN and (D) PPARG and SREBP1 transcription factors were evaluated by RT-qPCR. The relative gene expression levels are shown normalized to their corresponding untreated controls. Data are expressed as the mean $\pm \operatorname{SEM}(n=4$ experiments). Significant differences: ${ }^{*} p<0.05,{ }^{* *} p<0.01$ and ${ }^{* * *} p<0.001$. Detailed information about the Western blotting can be found in Figure S6. Abbreviations: p-JNK, phospho-Jun N-terminal Kinase; p-ERK, phospho-extracellular signal-regulated kinase, p-p38, phospho- 338 mitogen-activated protein kinase, $\mathrm{p}-\mathrm{AKT}$, phospho- Protein kinase B; $\beta$-Actin, beta-actin; CPT1A, Carnitine palmitoyltransferase IA; FABP4, fatty acid-binding protein 4; FASN, Fatty acid synthase; SREBP1, Sterol regulatory element-binding transcription factor 1; PPARG, Peroxisome proliferator-activated receptor gamma; \% over control: values are calculated as percentage over untreated cells at time 0; kDa, Kilodaltons: RT-qPCR, reverse transcription quantitative PCR. 
To test whether these activated pathways were implicated in the control of the lipid metabolism genes after sTWEAK/Fn14 engagement, we pharmacologically inhibited ERK1/2 and AKT signaling in PC-3 cells. We observed that the expression of genes implicated in lipolysis (CPT1), lipid transport (FABP4) and de novo lipogenesis (FASN) were abolished only by the ERK1/2 inhibitor (Figure $5 C$ ), whereas the expression of the gene transcription regulators SREBP-1 and PPARG were significantly regulated the transcriptional level by both ERK1/2 (PD184352) and AKT (MK2206) inhibitors (Figure 5D).

sTWEAK/Fn14 binding can also activate NF-kB canonical and noncanonical pathways in PC-3 cells (and in LNCaP cells; Figure S4); however, specific inhibitor experiments performed in PC-3 cells showed no implication of these pathways in the lipid metabolism (Figure S5).

\section{Discussion}

PCa remains one of the most prevalent cancers in men, and the accurate staging and detection of recurrent and metastatic disease remains a clinical challenge. Once detected, PCa can be treated with different approaches, including active surveillance, or with more aggressive surgical, radiation-based and other focal therapies [28]. All therapies used may have an impact on health-related quality of life. Most PCa cases follow an indolent course that would not threaten the duration or quality of life of patients, but tumor evolution is difficult to predict, as little is known about the factors that influence the onset of PCa or why some tumors progress from indolent to aggressive disease states.

Evaluating changes in metabolic biomarkers in relation to PCa diagnosis might be important to distinguish their contribution to cancer development. In this sense, we observed that hyperinsulinemia and insulin resistance measured by the HOMA-IR index were associated with PCa, as previously reported by others [29]. Hyperinsulinemia has also been reported as a risk factor in PCa development [8,30]. Additionally, the creatinine levels were higher in patients with $\mathrm{PCa}$, but no association with the risk of developing PCa was observed in our cohort, although this association has been reported [31].

Here, we report that the serum levels of the proinflammatory cytokine TWEAK, which has previously been related to glucose metabolism [18], were lower in patients with PCa than in healthy controls. Interestingly, the reduced levels of sTWEAK together with higher levels of PSA, a higher HOMA-IR index and higher LDL cholesterol levels were associated with PCa in our cohort, indicating that TWEAK might be an important component of this pathology. As far as we know, there is no other evidence to suggest that serum sTWEAK concentrations are linked to PCa.

TWEAK is known to be present in the PCa microenvironment, particularly in semen, where its levels are reduced in high- versus low-risk patients [26]. Additionally, the reduced concentration of sTWEAK was inversely related to the expression of the Fn14 gene, a relationship that could explain an active ligand-receptor activity in situ [26]. The tumor metabolic changes induced by alterations of inflammatory cytokines in PCa have been poorly described. Accordingly, the study of PCa cancer cell metabolism, which is experiencing a renaissance in interest [32], under the effect of sTWEAK, might cast some light on the mechanistic understanding of tumorigenesis. Prostate tumor metabolism has been described to be highly lipogenic and lipolytic [33], with relatively low glucose uptake and glycolysis rates mainly in the early stages of PCa [34] compared with other solid tumors [5].

To test the possible metabolic actions of this cytokine on PCa cells, we used two established in vitro models of PCa disease: PC-3 cells (androgen-independent and invasive) and LNCaP cells (androgen-dependent and noninvasive). As a control for these studies, we used nontumorigenic RWPE-1 prostate epithelial cells [33]. LNCaP and PC3 cell lines can be models for different stages of prostate cancer. Our analysis of glucose metabolism-related genes in the studied cell lines revealed the reduced gene expression in the "preparatory phase" of glycolysis after sTWEAK treatment. By contrast, in PC-3 cells, sTWEAK administration caused a significant upregulation of genes of the "payoff 
phase", PDHA1 and PKM2, which may render pyruvate as a substrate and favoring its entry into the mitochondria for conversion into citrate and, ultimately, fatty acids. A possible explanation for the differences in the expression of preparatory- and payoff-phase genes in PC-3 cells might be the use of glycerol for dihydroxyacetone phosphate synthesis and conversion to pyruvate (Figure 6).

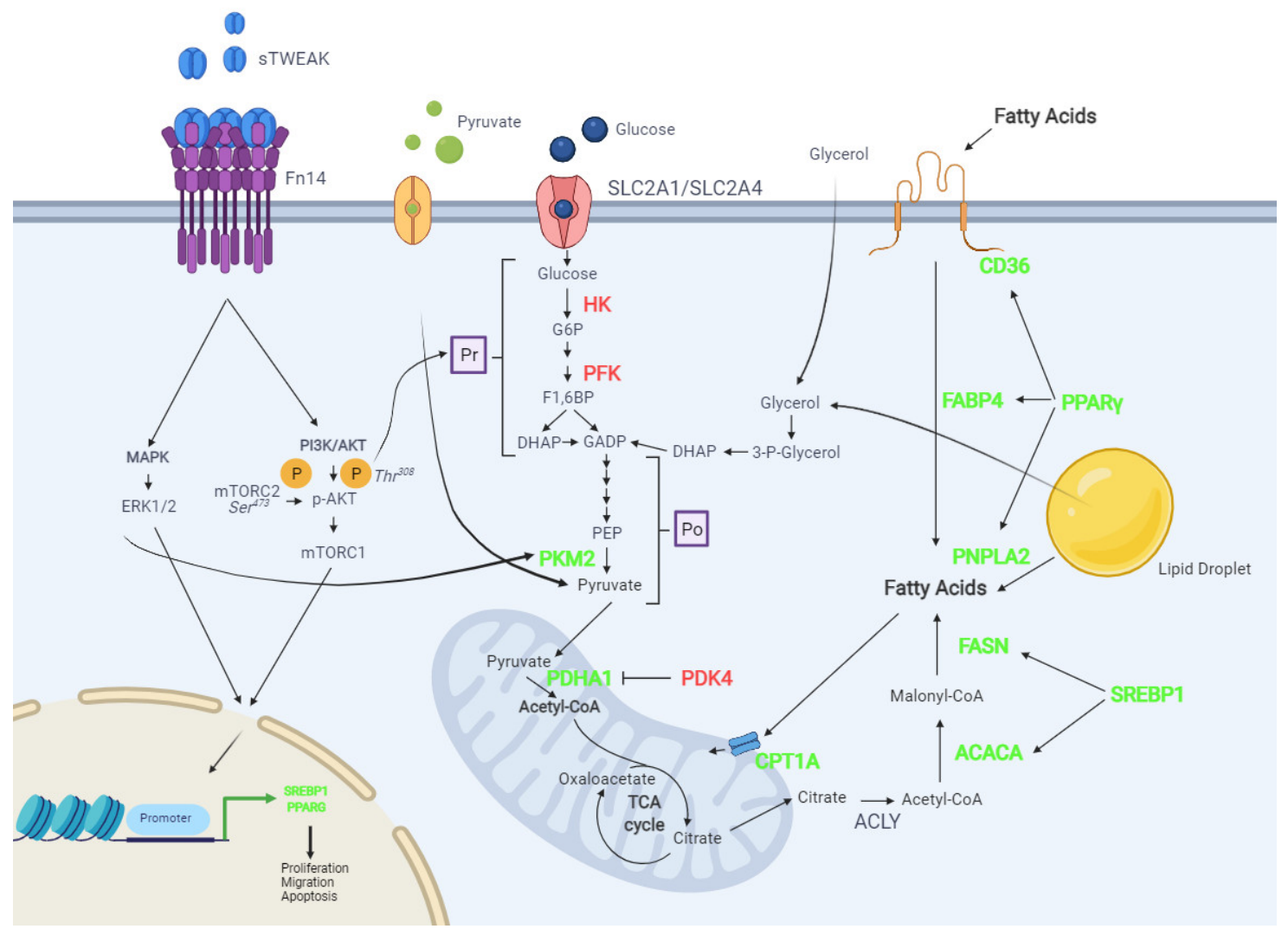

Figure 6. A scheme depicting the possible effects of the TWEAK/Fn14 axis on lipid and glucose metabolic pathways in PCa cells. TWEAK/Fn14 engagement phosphorylates AKT in ser473. PI3K-AKT-mTORC1 signaling is a key factor in controlling lipid and glucose metabolism. pAKT can regulate SREBP1 translocation to the nucleus, increasing ACLY, ACACA and FASN expression, potentiating de novo lipogenesis. AKT phosphorylation can also regulate PPARG, thus increasing the expression of the lipase PNPLA2, which catalyzes the breakdown of the ester bonds of fatty acids and activates lipolysis. Lipid entry and transport is also upregulated by PPARG through CD36 and FABP4 expression. CPT1A upregulation by sTWEAK would indicate that lipid oxidation in the mitochondria is boosted. De novo lipogenesis begins with citrate, which is generated by the conversion of pyruvate to acetyl-CoA mediated by an increase in the expression of PDHA1 and a decrease in PDK4 inhibitor expression, both actions regulated by sTWEAK/Fn14 engagement. Phosphorylation of ERK1/2 by sTWEAK/Fn14 engagement can regulate SREBP1 and PPARG and can also increase intracellular pyruvate concentrations by phosphorylating PKM2 and increasing its expression. The preliminary phase of glycolysis might be reduced, since $H K$ and PFK expression is downregulated. Exogenous pyruvate can also be taken up. Glycerol may be an alternative source for pyruvate. Color pattern: green color represents upregulated genes and red downregulated genes; Created with BioRender.com. Abbreviations: PI3K, Phosphoinositide 3-kinase; AKT, protein kinase B; p-AKT, phosphorylated protein kinase B; MAPK, mitogen-activated protein kinase; ERK, extracellular signal-regulated kinase; TF, transcription factor; SLC2A1, glucose transporter 1, SLC2A4, glucose transporter 4; Pr, preparatory phase; HK, hexokinase; PFK, Phosphofructokinase; G6P, Glucose 6-phosphate; F1,6BP, Fructose-1,6-Biphosphate; DHAP, Dihydroxyacetone phosphate; GADP, glyceraldehyde-3-phosphate; Po, Payoff phase; PEP, phosphoenolpyruvate; PKM2, Pyruvate kinase 2; PDHA1, Pyruvate dehydrogenase; PDK4, Pyruvate dehydrogenase lipoamide kinase isozyme 4; TCA, tricarboxylic acid; ACLY, ATP citrate synthase; ACACA, Acetyl-CoA Carboxylase Alpha; SREBP1, Sterol regulatory element-binding transcription factor 1; FASN, Fatty acid synthase; PNPLA2, patatin-like phospholipase 2; PPAR $\gamma$, Peroxisome proliferator-activated receptor gamma; FABP4, fatty acid-binding protein 4; CD36, cluster of differentiation 36; CPT1A, Carnitine palmitoyltransferase IA; 3-P-Glycerol, 3-phosphoglycerol. 
Metabolic imaging of PCa tumors with labeled pyruvate [35] has been more useful than glucose, because PCa tumors show a relatively low glucose uptake [36]. Pyruvate can also be obtained by PCa cells through glycerol metabolism, because a higher activity of mitochondrial glycerol-3-phosphatedehydrogenase, which regulates cytosolic glycerol3-phosphate (G3P), has been detected in PCa [37]. The use of glycerol by PCa cells has been indirectly demonstrated, because the enzyme monoacylglycerol lipase (MAGL), which converts monoacylglycerol into glycerol, has been shown to be upregulated in more aggressive PCa cell lines [38]. Glycerol can also be externally provided to tumor cells by PCa adipose tissue, as adipocytes treated with PCa-conditioned media increase their levels of PNPLA2 and secrete large amounts of free glycerol that may enter the PCa cells, fueling lipid metabolism [39] (Figure 6).

Of note, although sTWEAK does not positively regulate genes implicated in glucose metabolism in the studied PCa cells, glucose uptake experiments have demonstrated a low basal glucose metabolism in PCa cells versus a dominant fatty acid uptake [40,41], suggesting that fatty acids intake should be envisaged as target for further therapeutic PCa treatment [42].

Deregulation of lipogenic enzymes in PCa correlates with worse prognosis and poor survival [43]. sTWEAK treatment of PC-3 cells upregulated de novo lipogenesis, as evidenced by an increase in $A C A C A$ and FASN gene expression. Both genes are involved in the conversion of mitochondrial-derived citrate to fatty acids. This was accompanied by an increase in the expression of master key lipid regulatory genes such as SREBP-1 and PPARG and by upregulation of the lipid membrane transporter gene CD36 and the cytoplasmic fatty acid-binding protein $F A B P 4$. Consistent with this finding, we found a concordant sTWEAK-induced increase in cellular lipids and enhanced fatty acid uptake. The upregulation of FASN expression by sTWEAK is particularly relevant, as it can act as an oncogene in $\mathrm{PCa}$ [44], and FASN protein overexpression has been linked to poor recurrence-free survival in PCa [45]. Fatty acid oxidation is also reported to be deregulated in cancer cells [46], and, in this scenario, sTWEAK might upregulate this lipolytic process by increasing CPT1 expression in the mitochondria and PNPLA2 expression in the cytoplasm, both reported to be deregulated in PCa [47,48].

The enhancing effect of sTWEAK on lipid metabolism gene expression in the PC-3 cellular model was mediated by binding to the Fn14 receptor, which was paralleled by an increase in the corresponding protein expression. Overall, our observations suggest that sTWEAK treatment in PC-3 cells stimulates cells to obtain energy by the oxidation of fatty acids. Therefore, since lipid metabolism may confer more aggressive properties to malignant cancers cells [49], sTWEAK may be potentiating an aggressive PCa progression.

Several signaling cascades activated by TWEAK/Fn14 are implicated in PCa, including the canonical and noncanonical NF-kB signaling pathways and MAPKs [50]. Of the three known MAPK pathways-extracellular signal-regulated kinases (ERKs), c-Jun N-terminal kinases (JNKs) and p38 kinases [51] —only ERKs and JNKs have been shown to be key metabolic regulators in malignant cells [27]. We found that TWEAK activates the ERK1/2 pathway in PCa cells, influencing the cellular metabolism. This effect could be mediated by phosphorylating PKM2 [27], which, in turn, can regulate SREBP-1 expression, a key regulator of lipid metabolism in several cancer types [52] (Figure 6).

Activation of the PI3K-AKT-mTORC1 pathway is important in the regulation of lipid and glucose metabolisms [53,54]. sTWEAK treatment of PCa cells phosphorylates AKT at serine473, known to be mediated by mTORC2 [55] (Figure 6). The mechanistic links between Akt and SREBP on the lipid metabolism are well-known [56]. Of note, the timing of activation differed between PC-3 and LNCaP, being a late event in the former. This distinctive signaling trait between the two PCa lines in the present study might, in part, explain the differences observed in lipid gene expression.

We are aware that cell line-based studies have inherent limitations, as they likely do not represent the heterogeneity observed in PCa, but they are nevertheless useful tools to explore the mechanisms underlying tumorigenesis and drug resistance. Future studies with 
patient-derived cancer models, which are more representative of the original tumor [57], will be needed as the next step to explore the mechanistic relationship between the cytokine TWEAK and lipid metabolism in PCa.

\section{Conclusions}

Our results lead us to conclude that reduced serum levels of sTWEAK are independently associated with an increased risk of PCa. Additionally, we established that the presence of sTWEAK in the PCa microenvironment contributes to PCa lipid metabolism by modulating the expression of lipid-related genes, thus potentiating an aggressive progression. Since the inhibition of TWEAK/Fn14 engagement can block this metabolic effect in vitro, targeting Fn14 might be a therapeutic strategy for the management of PCa.

Supplementary Materials: The following are available online at https://www.mdpi.com/article/10 .3390 / cancers13184688/s1, Figure S1: FN14 gene expression in all the studied PCa cell lines (PC-3, LNCaP and RWPE-1). Figure S2: sTWEAK on the protein expression of CPT1A, FABP4 and FASN. Figure S3: Complete Western blotting results, referring to Figure 3. Figure S4: Effect of sTWEAK stimulation on several signaling pathways in the PCa cell models. Figure S5: PC-3 cells were treated with inhibitors of noncanonical and canonical NK-kB signaling. Figure S6: Complete Western blotting results, referring to Figure 5.

Author Contributions: Conceptualization, M.R.C., J.S.-T. and X.R.-P.; methodology, A.A.-C. and M.A.-S.; formal analysis, M.R.C. and A.A.-C.; writing-review and editing, M.R.C., X.R.-P., J.S.-T. and A.A.-C. and funding acquisition, M.R.C. and X.R.-P. All authors have read and agreed to the published version of the manuscript.

Funding: This study was founded by Instituto de Salud Carlos III through projects PI17/00877 and PI20/00418 (co-funded by the European Regional Development Fund/European Social Fund, “A way to make future" / "Investing in your future"). No payment was received to write this article by a pharmaceutical company or other agency.

Institutional Review Board Statement: This study was conducted according to the guidelines of the Declaration of Helsinki and approved by the Institutional Review Board of Insitut d'Investigació Sanitària Pere Virgili (Ref. CEIM 171/2017; Ref. CEIM205/2020).

Informed Consent Statement: Informed consent was obtained from all the subjects involved in the study.

Data Availability Statement: The data presented in this study are available on request from the corresponding authors.

Acknowledgments: We wish to acknowledge the patients enrolled in this study for their participation and the IISPV BioBanc (B.0000853 + B.0000854) integrated in the Spanish National Biobanks Platform (PT13/0010/0029 and PT13/0010/0062) for its collaboration.

Conflicts of Interest: The authors declare that they have no competing interests.

\section{References}

1. Sung, H.; Ferlay, J.; Siegel, R.L.; Laversanne, M.; Soerjomataram, I.; Jemal, A.; Bray, F. Global Cancer Statistics 2020: GLOBOCAN Estimates of Incidence and Mortality Worldwide for 36 Cancers in 185 Countries. CA Cancer J. Clin. 2021, 71, 209-249. [CrossRef] [PubMed]

2. Epstein, J.I.; Egevad, L.; Amin, M.B.; Delahunt, B.; Srigley, J.R.; Humphrey, P.A. The 2014 International Society of Urological Pathology (ISUP) consensus conference on Gleason grading of prostatic carcinoma: Definition of grading patterns and proposal for a new grading system. Am. J. Surg. Pathol. 2016, 40, 244-252. [CrossRef]

3. Koundouros, N.; Poulogiannis, G. Reprogramming of fatty acid metabolism in cancer. Br. J. Cancer 2020, 122, 4-22. [CrossRef] [PubMed]

4. Fernández, L.P.; Gómez de Cedrón, M.; Ramírez de Molina, A. Alterations of Lipid Metabolism in Cancer: Implications in Prognosis and Treatment. Front. Oncol. 2020, 10, 577420. [CrossRef] [PubMed]

5. Bader, D.A.; McGuire, S.E. Tumour metabolism and its unique properties in prostate adenocarcinoma. Nat. Rev. Urol. 2020, 17, 214-231. [CrossRef] [PubMed]

6. Zadra, G.; Photopoulos, C.; Loda, M. The fat side of prostate cancer. Biochim. Biophys. Acta 2013, 1831, 1518-1532. [CrossRef]

7. Saboori, S.; Rad, E.Y.; Birjandi, M.; Mohiti, S.; Falahi, E. Serum insulin level, HOMA-IR and prostate cancer risk: A systematic review and meta-analysis. Diabetes Metab. Syndr. Clin. Res. Rev. 2019, 13, 110-115. [CrossRef] 
8. Nandeesha, H. Insulin: A novel agent in the pathogenesis of prostate cancer. Int. Urol. Nephrol. 2009, 41, 267-272. [CrossRef]

9. Sfanos, K.S.; De Marzo, A.M. Prostate cancer and inflammation: The evidence Karen. Histopathology 2012, 60, 199-215. [CrossRef]

10. Maecker, H.; Varfolomeev, E.; Kischkel, F.; Lawrence, D.; LeBlanc, H.; Lee, W.; Hurst, S.; Danilenko, D.; Li, J.; Filvaroff, E.; et al. TWEAK attenuates the transition from innate to adaptive immunity. Cell 2005, 123, 931-944. [CrossRef]

11. Chicheportiche, Y.; Bourdon, P.R.; Xu, H.; Hsu, Y.M.; Scott, H.; Hession, C.; Garcia, I.; Browning, J.L. TWEAK, a new secreted ligand in the tumor necrosis factor family that weakly induces apoptosis. J. Biol. Chem. 1997, 272, 32401-32410. [CrossRef]

12. Winkles, J.A. The TWEAK-Fn14 cytokine-receptor axis: Discovery, biology and therapeutic targeting. Nat. Rev. Drug Discov. 2008, 7, 411-425. [CrossRef] [PubMed]

13. Wiley, S.R.; Winkles, J.A. TWEAK, a member of the TNF superfamily, is a multifunctional cytokine that binds the TweakR/Fn14 receptor. Cytokine Growth Factor Rev. 2003, 14, 241-249. [CrossRef]

14. Narita, S.N.; Tsuchiya, N.; Saito, M.; Inoue, T.; Kumazawa, T.; Yuasa, T.; Nakamura, A.; Habuchi, T. CandidateGenes Involved in Enhanced Growth of Human Prostate Cancer Under High Fat Feeding. Identified by Microarray Analysis. Prostate 2008, 68, 321-335. [CrossRef] [PubMed]

15. Huang, M.; Narita, S.; Tsuchiya, N.; Ma, Z.; Numakura, K.; Obara, T.; Tsuruta, H.; Saito, M.; Inoue, T.; Horikawa, Y.; et al. Overexpression of Fn14 promotes androgen-independent prostate cancer progression through MMP-9 and correlates with poor treatment outcome. Carcinogenesis 2011, 32, 1589-1596. [CrossRef]

16. Ruiz-Plazas, X.; Rodríguez-Gallego, E.; Alves, M.; Altuna-Coy, A.; Lozano-Bartolomé, J.; Portero-Otin, M.; García-Fontgivell, J.F.; Martínez-González, S.; Segarra, J.; Chacón, M.R. Biofluid quantification of TWEAK/Fn14 axis in combination with a selected biomarker panel improves assessment of prostate cancer aggressiveness. J. Transl. Med. 2019, 17, 307. [CrossRef] [PubMed]

17. Ruiz-Plazas, X.; Altuna-Coy, A.; Alves-Santiago, M.; Vila-Barja, J.; García-Fontgivell, J.F.; Martínez-González, S.; Segarra-Tomás, J.; Chacón, M.R. Liquid biopsy-based exo-oncomirnas can predict prostate cancer aggressiveness. Cancers 2021, 13, 250. [CrossRef] [PubMed]

18. Vendrell, J.; Chacón, M.R. TWEAK: A new player in obesity and diabetes. Front. Immunol. 2013, 4, 488. [CrossRef]

19. Lozano-Bartolomé, J.; Llauradó, G.; Rodriguez, M.M.; Fernandez-Real, J.M.; Garcia-Fontgivell, J.F.; Puig, J.; Maymó-Masip, E.; Vendrell, J.; Chacón, M.R. Reduced circulating levels of sTWEAK are associated with NAFLD and may affect hepatocyte triglyceride accumulation. Int. J. Obes. 2016, 40, 1337-1345. [CrossRef]

20. Preston, M.A.; Batista, J.L.; Wilson, K.M.; Carlsson, S.V.; Gerke, T.; Sjoberg, D.D.; Dahl, D.M.; Sesso, H.D.; Feldman, A.S.; Gann, P.H.; et al. Baseline prostate-specific antigen levels in midlife predict lethal prostate cancer. J. Clin. Oncol. 2016, 34, $2705-2711$. [CrossRef]

21. Chen, S.; Liu, J.; Yang, M.; Lai, W.; Ye, L.; Chen, J.; Hou, X.; Ding, H.; Zhang, W.; Wu, Y.; et al. Fn14, a downstream target of the TGF- $\beta$ signaling pathway, regulates fibroblast activation. PLoS ONE 2015, 10, e0143802. [CrossRef] [PubMed]

22. Pereira, S.S.; Monteiro, M.P.; Costa, M.M.; Ferreira, J.; Alves, M.G.; Oliveira, P.F.; Jarak, I.; Pignatelli, D. MAPK/ERK pathway inhibition is a promising treatment target for adrenocortical tumors. J. Cell. Biochem. 2019, 120, 894-906. [CrossRef]

23. Tee, S.S.; Suster, I.; Truong, S.; Jeong, S.; Eskandari, R.; DiGialleonardo, V.; Alvarez, J.A.; Aldeborgh, H.N.; Keshari, K.R. Targeted AKT inhibition in prostate cancer cells and spheroids reduces aerobic glycolysis and generation of hyperpolarized [1-13C] lactate. Mol. Cancer Res. 2018, 16, 453-460. [CrossRef]

24. Sun, Y.; St Clair, D.K.; Xu, Y.; Crooks, P.A.; St Clair, W.H. A NADPH oxidase-dependent redox signaling pathway mediates the selective radiosensitization effect of parthenolide in prostate cancer cells. Cancer Res. 2010, 70, 2880-2890. [CrossRef] [PubMed]

25. Henry, K.L.; Kellner, D.; Bajrami, B.; Anderson, J.E.; Beyna, M.; Bhisetti, G.; Cameron, T.; Capacci, A.G.; Bertolotti-Ciarlet, A.; Feng, J.; et al. CDK12-mediated transcriptional regulation of noncanonical NF-kB components is essential for signaling. Sci. Signal. 2018, 11, eaam8216. [CrossRef]

26. Vendrell, J.; Maymó-Masip, E.; Tinahones, F.; García-España, A.; Megia, A.; Caubet, E.; García-Fuentes, E.; Chacón, M.R. Tumor necrosis-like weak inducer of apoptosis as a proinflammatory cytokine in human adipocyte cells: Up-regulation in severe obesity is mediated by inflammation but not hypoxia. J. Clin. Endocrinol. Metab. 2010, 95, 2983-2992. [CrossRef] [PubMed]

27. Papa, S.; Choy, P.M.; Bubici, C. The ERK and JNK pathways in the regulation of metabolic reprogramming. Oncogene 2019, 38, 2223-2240. [CrossRef]

28. Cooperberg, M.R.; Carroll, P.R.; Klotz, L. Active surveillance for prostate cancer: Progress and promise. J. Clin. Oncol. 2011, 29, 3669-3676. [CrossRef]

29. Di Sebastiano, K.M.; Pinthus, J.H.; Duivenvoorden, W.C.M.; Mourtzakis, M. Glucose impairments and insulin resistance in prostate cancer: The role of obesity, nutrition and exercise. Obes. Rev. 2018, 19, 1008-1016. [CrossRef]

30. Hammarsten, J.; Högstedt, B. Hyperinsulinaemia: A prospective risk factor for lethal clinical prostate cancer. Eur. J. Cancer 2005, 41, 2887-2895. [CrossRef]

31. Weinstein, J.E.; Mackrain, K.; Stolzenberg-Solomon, R.-Z.; Selhub, J.; Virtamo, J.; Albanes, D. Serum creatinine and prostate cancer risk in a prospective study. Cancer Epidemiol. Biomark. Prev. 2009, 18, 2643-2649. [CrossRef] [PubMed]

32. Harris, A.L. Development of cancer metabolism as a therapeutic target: New pathways, patient studies, stratification and combination therapy. Br. J. Cancer 2020, 122, 1-3. [CrossRef] [PubMed]

33. Butler, L.M.; Centenera, M.M.; Swinnen, J.V. Androgen control of lipid metabolism in prostate cancer: Novel insights and future applications. Endocr. Relat. Cancer 2014, 23, R219-R227. [CrossRef] 
34. Jadvar, H. PET of glucose metabolism and cellular proliferation in prostate cancer. J. Nucl. Med. 2016, 57, 25S-29S. [CrossRef] [PubMed]

35. Beauregard, J.M.; Williams, S.G.; Degrado, T.R.; Roselt, P.; Hicks, R.J. Pilot comparison of 18F-fluorocholine and 18F- fluorodeoxyglucose PET/CT with conventional imaging in prostate cancer. J. Med. Imaging Radiat. Oncol. $2010,54,325-332$. [CrossRef] [PubMed]

36. Aggarwal, R.; Vigneron, D.B.; Kurhanewicz, J.; Francisco, S. Hyperpolarized 1-[13C]-Pyruvate Magnetic Resonance Imaging Detects an Early Metabolic Response to Androgen Ablation Therapy in Prostate Cancer. Eur. Urol. 2017, 72, 1028-1029. [CrossRef]

37. Pecinová, A.; Alán, L.; Brázdová, A.; Vrbacký, M.; Pecina, P.; Drahota, Z.; Houštěk, J.; Mráček, T. Role of Mitochondrial Glycerol-3-Phosphate Dehydrogenase in Metabolic Adaptations of Prostate Cancer. Cells 2020, 9, 1764. [CrossRef]

38. Nomura, D.K.; Lombardi, D.P.; Chang, J.W.; Niessen, S.; Ward, A.M.; Long, J.Z.; Hoover, H.H.; Cravatt, B.F. Monoacylglycerol lipase exerts dual control over endocannabinoid and fatty acid pathways to support prostate cancer. Chem. Biol. 2011, 18, 846-856. [CrossRef]

39. Diedrich, J.D.; Rajagurubandara, E.; Herroon, M.K.; Mahapatra, G.; Hüttemann, M.; Podgorski, I. Bone marrow adipocytes promote the warburg phenotype in metastatic prostate tumors via HIF-1 $\alpha$ activation. Oncotarget 2016, 7, 64854-64877. [CrossRef]

40. Liu, Y.; Zuckier, L.S.; Ghesani, N.V. Dominant uptake of fatty acid over glucose by prostate cells: A potential new diagnostic and therapeutic approach. Anticancer Res. 2010, 30, 369-374.

41. Dueregger, A.; Schöpf, B.; Eder, T.; Höfer, J.; Gnaiger, E.; Aufinger, A.; Kenner, L.; Perktold, B.; Ramoner, R.; Klocker, H.; et al. Differential utilization of dietary fatty acids in benign and malignant cells of the prostate. PLoS ONE 2015, 10, e0135704. [CrossRef]

42. Liu, Y. Fatty acid oxidation is a dominant bioenergetic pathway in prostate cancer. Prostate Cancer Prostatic Dis. 2006, 9, 230-234. [CrossRef]

43. Dang, Q.; Chen, Y.-A.; Hsieh, J.-T. The dysfunctional lipids in prostate cancer. Am. J. Clin. Exp. Urol. 2019, 7, $273-280$.

44. Loda, M.; Migita, T.; Ruiz, S.; Fornari, A.; Fiorentino, M.; Priolo, C.; Zadra, G.; Inazuka, F.; Grisanzio, C.; Palescandolo, E.; et al. Fatty acid synthase: A metabolic enzyme and candidate oncogene in prostate cancer. J. Natl. Cancer Inst. 2009, 101, 519-532. [CrossRef]

45. Cao, Z.; Xu, Y.; Guo, F.; Chen, X.; Ji, J.; Xu, H.; He, J.; Yu, Y.; Sun, Y.; Lu, X.; et al. FASN Protein Overexpression Indicates Poor Biochemical Recurrence-Free Survival in Prostate Cancer. Dis. Markers 2020, 2020, 3904947. [CrossRef] [PubMed]

46. Rhodes, D.R.; Yu, J.; Shanker, K.; Deshpande, N.; Varambally, R.; Ghosh, D.; Barrette, T.; Pandey, A.; Chinnaiyan, A.M. ONCOMINE: A Cancer Microarray Database and Integrated Data-Mining Platform. Neoplasia 2004, 6, 1-6. [CrossRef]

47. Schlaepfer, I.R.; Rider, L.; Rodrigues, L.U.; Gijón, M.A.; Colton, T.; Romero, L.; Cimic, A.; Sirintrapun, S.J.; Glodé, L.M.; Robert, H.; et al. Lipid catabolism via CPT1 as a therapeutic target for prostate cancer. Mol. Cancer Ther. 2014, 13, 2361-2371. [CrossRef]

48. Mitra, R.; Le, T.T.; Gorjala, P.; Goodman, O.B. Positive regulation of prostate cancer cell growth by lipid droplet forming and processing enzymes DGAT1 and ABHD5. BMC Cancer 2017, 17, 631. [CrossRef]

49. Luo, X.; Cheng, C.; Tan, Z.; Li, N.; Tang, M.; Yang, L.; Cao, Y. Emerging roles of lipid metabolism in cancer metastasis. Mol. Cancer 2017, 16, 1-10. [CrossRef]

50. Yin, J.; Morrissey, C.; Barrett, B.; Corey, E.; Ylaya, K.; Hewitt, S.; Fang, L.; Tillman, H.; Lake, R.; Vessella, R.; et al. AR-Regulated TWEAK-FN14 Pathway Promotes Prostate Cancer Bone Metastasis. Cancer Res. 2014, 74, 4306-4317. [CrossRef]

51. Cargnello, M.; Roux, P.P. Activation and Function of the MAPKs and Their Substrates, the MAPK-Activated Protein Kinases. Microbiol. Mol. Biol. Rev. 2011, 75, 50-83. [CrossRef] [PubMed]

52. Tao, T.; Su, Q.; Xu, S.; Deng, J.; Zhou, S.; Zhuang, Y.; Huang, Y.; He, C.; He, S.; Peng, M.; et al. Down-regulation of PKM2 decreases FASN expression in bladder cancer cells through AKT/mTOR/SREBP-1c axis. J. Cell. Physiol. 2019, 234, 3088-3104. [CrossRef] [PubMed]

53. Hoxhaj, G.; Manning, B. The PI3K-AKT network at the interface of oncogenic signalling and cancer metabolism. Nat Rev Cancer 2020, 20, 74-88. [CrossRef]

54. Götting, I.; Jendrossek, V.; Matschke, J. A new twist in protein kinase b/akt signaling: Role of altered cancer cell metabolism in akt-mediated therapy resistance. Int. J. Mol. Sci. 2020, 21, 8563. [CrossRef]

55. Manning, B.D.; Toker, A. AKT/PKB Signaling: Navigating the Network. Cell 2017, 169, 381-405. [CrossRef] [PubMed]

56. Krycer, J.R.; Sharpe, L.J.; Luu, W.; Brown, A.J. The Akt-SREBP nexus: Cell signaling meets lipid metabolism. Trends Endocrinol. Metab. 2010, 21, 268-276. [CrossRef]

57. Namekawa, T.; Ikeda, K.; Horie-Inoue, K.; Inoue, S. Application of Prostate Cancer Models for Preclinical Study: Advantages and Limitations of Cell Lines, Patient-Derived Xenografts, and Three-Dimensional Culture of Patient-Derived Cells. Cells 2019, 8, 74. [CrossRef] [PubMed] 